\title{
Crisis management during anaesthesia: anaphylaxis and allergy
}

\author{
M Currie, R K Kerridge, A K Bacon, J A Williamson
}

Qual Saf Health Care 2005;14:e19 (http://www.qshc.com/cgi/content/full/14/3/e19). doi: 10.1136/qshc.2002.004465

See end of article for authors' affiliations

.....................

Correspondence to: Professor W B Runciman, President, Australian Patient Safety Foundation GPO Box 400, Adelaide, South Australia, 5001, Australia; research@apsf. net.au

Accepted 12 January 2005
Background: Anaphylactic and anaphylactoid reactions during anaesthesia are a major cause for concern for anaesthetists. However, as individual practitioners encounter such events so rarely, the rapidity with which the diagnosis is made and appropriate management instituted varies considerably.

Objectives: To examine the role of a previously described core algorithm "COVER ABCD-A SWIFT $\mathrm{CHECK}^{\prime \prime}$, supplemented by a specific sub-algorithm for anaphylaxis, in the management of severe allergic reactions occurring in association with anaesthesia.

Methods: The potential performance of this structured approach for each of the relevant incidents among the first 4000 reported to the Australian Incident Monitoring Study (AIMS) was compared with the actual performance as reported by the anaesthetists involved.

Results: There were 148 allergic reactions among the first 4000 incidents reported to AIMS. It was considered that, properly applied, the structured approach would have led to a quicker and/or better resolution of the problem in $30 \%$ of cases, and would not have caused harm had it been applied in all of them.

Conclusion: An increased awareness of the diverse clinical manifestations of allergy seen in anaesthetic practice, together with the adoption of a structured approach to management should improve and standardise the treatment and improve follow up of patients suspected of having suffered a significant allergic reaction under anaesthesia.
A naphylactic and anaphylactoid reactions during anaesthesia are a major cause for concern for anaesthetists. As they occur only once in every 5-10 000 anaesthetics, ${ }^{12}$ individual anaesthetists are likely to encounter only a few cases in their working lifetimes. The possibility of anaphylaxis or an allergic reaction must be specifically considered and appropriate steps taken immediately whenever sudden hypotension or bronchospasm occurs, when there is difficulty breathing or swallowing, or when a skin or mucosal swelling is detected. However, as anaesthetists encounter such events so rarely, the rapidity with which the diagnosis is made and appropriate management instituted varies considerably. ${ }^{3}$

For this reason, the role of a structured approach in diagnosing and managing anaphylaxis and severe allergic reactions was examined. This paper builds on a previous report on anaphylaxis. ${ }^{3}$ In 1993, a "core" crisis management algorithm, represented by the mnemonic COVER ABCD-A SWIFT CHECK (the $\mathrm{AB}$ precedes COVER for the nonintubated patient), was proposed as the basis for a systematic approach to any crisis during anaesthesia where it is not immediately obvious what should be done, or where actions taken have failed to remedy the situation. ${ }^{4}$ This was validated against the first 2000 incidents reported to the original Australian Incident Monitoring Study (AIMS). AIMS is an ongoing study which involves the voluntary, anonymous reporting of any unintended incident which reduced, or could have reduced, the safety margin for a patient. ${ }^{5}$ It was concluded that if this algorithm had been correctly applied, a functional diagnosis would have been reached within $40-60$ seconds in $99 \%$ of applicable incidents, and the learned sequence of actions recommended by the COVER portion would have led to appropriate steps being taken to handle the $60 \%$ of problems relevant to this portion of the algorithm. ${ }^{4}$ However, this study also showed that the $40 \%$ of problems represented by the remainder of the algorithm,
ABCD-A SWIFT CHECK, were not always promptly diagnosed or appropriately managed..$^{4-6}$ It was decided that it would be useful, for these remaining problems, to develop a set of sub-algorithms in an easy to use crisis management manual. ${ }^{7}$ This study reports on the potential place of the COVER ABCD-A SWIFT CHECK algorithm in the diagnosis and initial management of anaphylaxis and severe allergic reactions, offers an outline of a specific crisis management sub-algorithm for these problems during anaesthesia, and provides an indication of the potential value of using this structured approach.

\section{METHODS}

Of the first 4000 incidents reported to the AIMS, those which made reference to any combination of anaphylaxis, allergy, hypotension, bronchospasm, skin flush or rash, urticaria, facial or airway oedema, or adrenaline administration, were extracted and analysed for relevance, presenting features, causes, diagnosis, management, and outcome.

The COVER ABCD-A SWIFT CHECK algorithm, described elsewhere in this set of articles, ${ }^{7}$ was applied to each relevant report to determine the stages at which the problem might have been diagnosed and to confirm that activating the COVER portion would have led to appropriate initial steps being taken. As anaphylaxis is not adequately dealt with by this algorithm, a specific sub-algorithm for anaphylaxis was developed (see figure) and its putative effectiveness was tested against the reports. How this was done is described elsewhere in this set of articles. ${ }^{7}$ The potential value of this structured approach (that is, the application of COVER ABCD-A SWIFT CHECK to the diagnosis and initial management of the problem, followed by the application of the anaphylaxis sub-algorithm) was assessed in the light of AIMS reports by comparing its potential effectiveness for each incident with that of the actual management, as recorded in each report. 


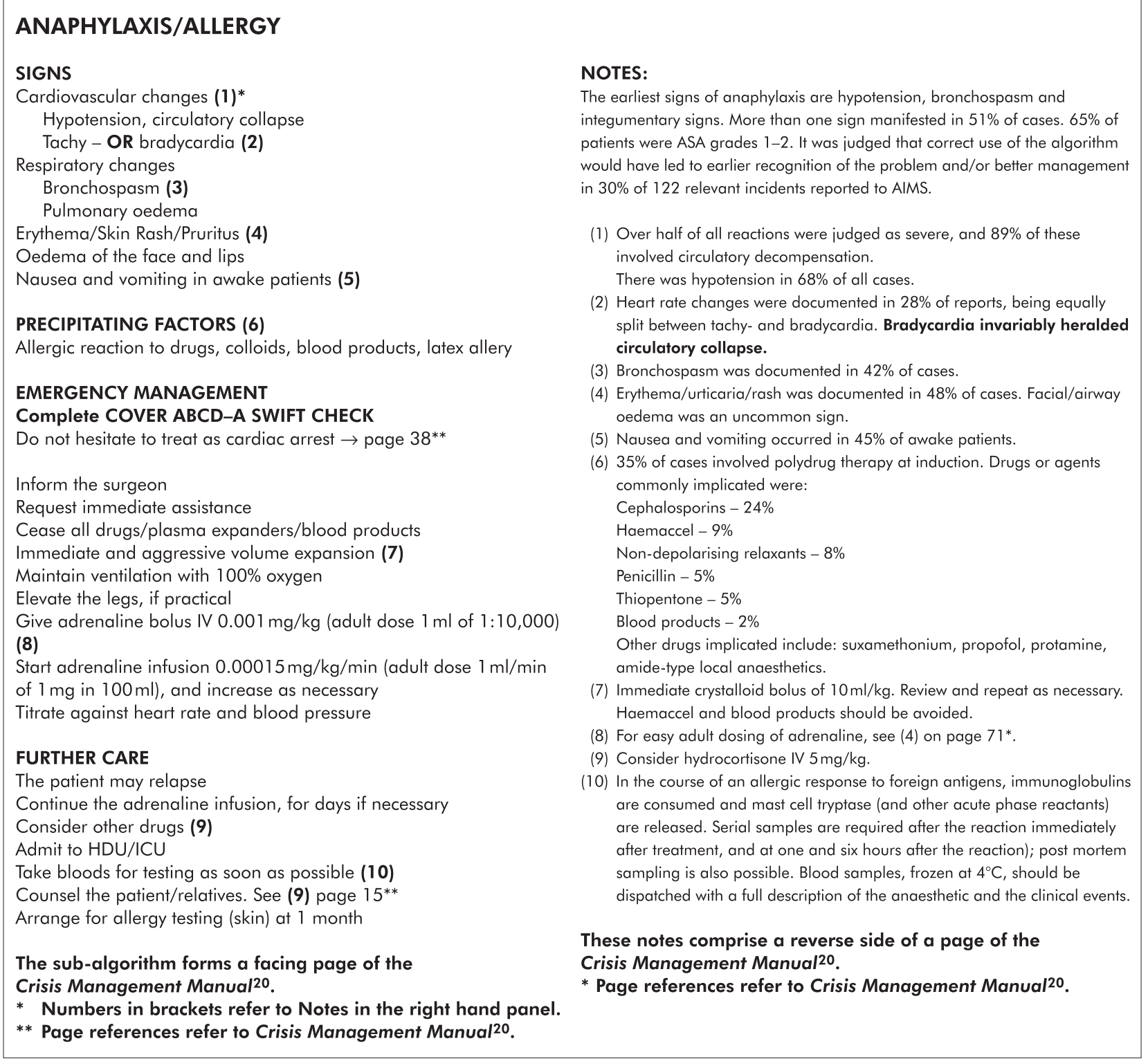

Figure 1 Anaphylaxis/allergy.

\section{RESULTS}

Of the first 4000 incidents reported to the AIMS, 225 were extracted for further study. Of these, 77 were excluded, because a cause other than allergy was considered more likely, leaving a total of 148 incidents for further analysis. Seventy six of these were judged to have been severe reactions (that is, life threatening and/or slow to resolve despite intervention). The presenting signs of these reactions are shown in table 1 . Among the severe cases, there were five deaths. In addition, 26 patients had an unplanned admission to a high dependency unit or a prolonged hospital stay, and in 10 cases the planned operative procedure was cancelled or abandoned.

Of the 148 reports, patients in ASA grades I and II featured in $65 \%$, and ASA grades III and IV in $35 \%$. This ratio was similar for "severe" and "less severe" reactions. There were 101 reports of hypotension (68\%), 62 reports of bronchospasm $(42 \%)$, of which three presented late, and 71 of skin or mucosal effects (48\%), of which six presented late. Heart rate
Table 1 Presenting signs of 76 severe anaphylactic reactions

\begin{tabular}{lc}
\hline Presenting signs & Number \\
\hline Hypotension & $31^{*}$ \\
Hypotension + skin signs & $21 \dagger$ \\
Hypotension + bronchospasm + skin signs & 12 \\
Hypotension + bronchospasm & 4 \\
Skin/mucosal signs & 4 \\
Bronchospasm & 2 \\
Bronchospasm + skin signs & 2 \\
Total & 76 \\
\hline & \\
*One also reported "difficult ventilation". & \\
†Two also reported "difficult ventilation". \\
Hypotension: includes a documented fall in systolic blood pressure, \\
unrecordable blood pressure, and impalpable pulses. \\
Bronchospasm: includes documented bronchospasm or difficulty with \\
ventilation. \\
Skin/mucosal signs: includes rash, urticaria, oedema, or swelling of any \\
part of the patient (including tongue and airway). \\
\hline
\end{tabular}


changes were documented in $28 \%$ of reports, and were equally split between tachycardia and bradycardia. Bradycardia invariably heralded circulatory collapse. Nausea and vomiting occurred in $45 \%$ of awake patients.

For all the reports of possible allergy, the management employed and its effectiveness was compared with that recommended in the literature. ${ }^{2-11}$ There was no evidence in the cases reported to the AIMS of a separate beneficial systemic effect of either antihistamine or steroid administration during the acute episode. There was ample evidence throughout the reports of the beneficial effect of adrenaline administration during the acute episode, on circulation, bronchospasm, and skin changes. Post-crisis care, documentation, and follow up of each incident were examined when recorded.

Of the 148 incidents, 122 incidents (64 "severe") were relevant to the core algorithm COVER ABCD-A SWIFT CHECK and the specific anaphylaxis sub-algorithm; 118 incidents occurred during induction, maintenance, or emergence from general anaesthesia and four occurred during regional anaesthesia, when the patient was breathing gas from an anaesthetic machine. In 35\% of cases, the reaction followed the use of several drugs at induction of anaesthesia. Agents commonly implicated in the reactions were cephalosporins (24\%), Haemaccel (9\%), non-depolarising relaxants $(8 \%)$, penicillin $(5 \%)$, thiopentone $(5 \%)$, and blood products (2\%). Other drugs implicated include: suxamethonium, propofol, protamine, and amide-type local anaesthetics.

When the COVER ABCD-A SWIFT CHECK algorithm was applied to each report, it was considered that a problem would have been detected in all "severe" cases at the Cl (circulation) stage of COVER or, failing that, at the Rl (review monitors) stage of COVER. The algorithm's performance was then evaluated for the 58 applicable less severe reactions. It was considered that if the diagnosis had not been made by the CHECK level of COVER that it should have been made when the A ("be Aware of Air and Allergy") of A SWIFT CHECK was considered. It was judged that the possible causes would be identified at the $\mathbf{D}$ (drugs) of the ABCD part of the algorithm, and that the actions recommended by COVER would have constituted appropriate immediate steps for anaphylaxis or a severe allergic reaction. It was also considered that carrying out the recommendations of the anaphylaxis sub-algorithm outlined in figure 1 would have constituted appropriate management in all cases. When the potential effectiveness of the structured approach represented by the COVER ABCD-A SWIFT CHECK algorithm and the sub-algorithm for anaphylaxis (see figure) was compared with that of the actual management, as documented in each of the 122 relevant incident reports, it was considered that, properly applied, the structured approach would have led to a quicker and/or better resolution of the problem in 36 cases (30\%). In these 36 reports there was significant delay in diagnosis or a delay in giving, or failure to give, adrenaline. For the 64 applicable "severe" reactions, the 15 incidents in which the algorithm would probably have outperformed the anaesthetist during the crisis were largely associated with delay in both the diagnosis of possible allergy and the administration of adrenaline. In none of the incidents was the application of the sub-algorithm considered harmful.

\section{DISCUSSION}

This review of 4000 incident reports reveals that up to $4 \%$ of the incidents reported were thought to have had an allergic aetiology, over half of which resulted in severe physiological insult. It has been estimated that such reactions are responsible for one life threatening situation in every 5-10 000 anaesthetics, and that once a reaction has started the mortality is $3-6 \% .^{2}{ }^{12}{ }^{13}$ Our analysis of the AIMS reports is consistent with these estimates.

The striking feature to emerge from our analysis was an apparent reluctance on the part of some anaesthetists in the AIMS reports to employ adrenaline as an appropriate early intervention. When what may generally be considered to be conservative doses of adrenaline were infused intravenously, these were remarkably effective, even in the most severe, multisystem reactions.

Although "allergy" was considered by the anaesthetist in the differential diagnosis of the cause of the incident in 112 reports, and in many cases may well have received some follow up, in only 27 reports was any allergy testing specifically mentioned in the narrative, and only 10 results of such testing were reported. Nine were positive. It would seem that a higher awareness of allergy testing needs to be promoted among anaesthetists; ${ }^{89}$ however, most reporters would have submitted their reports without waiting for the results of allergy testing, even if it had been arranged. The drugs implicated in the incident reports differ somewhat from those implicated in the literature. Overall during medical and surgical procedures, muscle relaxants have been shown to be responsible for $60-70 \%$ of cases, latex for $10-20 \%$, antibiotics for $5-20 \%$, colloids and induction agents for about $3-5 \%$ each. ${ }^{2-12}$ Latex seems to be becoming an increasingly frequent problem. ${ }^{14}$ In the AIMS reports, muscle relaxants appear to have been implicated much less commonly, and antibiotics more commonly than in most series.

All patients suspected of having had an allergic reaction should have plasma-histamine, tryptase, and specific IGE concentrations determined at the time of the reaction and, again, at one and six hours after the reaction. Skin tests should be conducted at six weeks. In a series of 789 patients, immune related (anaphylactic) reactions were implicated in two thirds of cases and non-immune related (anaphylactoid) reactions in one third, with anaphylactic reactions generally being more severe. ${ }^{12}$

In summary, anaesthetists should always think of an anaphylactic or anaphylactoid reaction with unexpected, sudden, or severe hypotension. Bronchospasm occurs less than half the time, under general anaesthesia, and skin and mucosal presentations may be late or obscured in the patient undergoing surgery. ${ }^{14}$ A high index of suspicion and early, aggressive therapy with adrenaline by intravenous injection is vital. An adrenaline infusion should be instituted as soon as possible, with titration against the heart rate and blood pressure. A very large dose may be required and the infusion may have to be maintained for hours and occasionally for days. ${ }^{11}$

Any diagnosis may be confounded by the use of multiple drugs at induction, some of which can cause hypotension in any event, the fact that the anaesthetist may be distracted by many events that may be taking place at the same time, and the fact that the patient may be extensively medicated.

As indicated above, all patients suspected of having had a reaction require plasma-histamine, tryptase, and specific IGE concentrations determined from the time of the reaction and during the next 24 hours. Skin tests should be conducted at about six weeks after the event.

Finally, it is important that a full explanation of what happened be given to the patient, that the event and the results of any tests should be documented in the anaesthetic record, and that the patient be given a letter to warn future anaesthetists. If a particular precipitating event was significant, or a particular action was useful in resolving the crisis, this should be clearly explained and documented. A permanent warning bracelet should be worn by the patient. Future use of muscle relaxants should be avoided, if at all 


\section{Key messages}

- There were 148 incidents that involved allergic reactions among the first 4000 reports to AIMS; 76 were severe and five were fatal.

- Twenty six cases had unplanned High Dependency Unit admission and in 10 cases the planned operative procedure was cancelled.

- Main presenting signs in the 76 severe cases were: hypotension alone (31), hypotension plus skin signs (21), hypotension plus bronchospasm plus skin changes (12).

- Hypotension was reported in $68 \%$ and bronchospasm in $42 \%$ of the cases.

- The $28 \%$ of heart rate changes were equally tachycardias and bradycardias.

- ASA grades I and II featured in $65 \%$ of the cases.

- Commonly implicated agents included cephalosporins (24\%), Haemaccel (9\%), non-depolarising relaxants $(8 \%)$ penicillin $(5 \%)$, thiopentone $(5 \%)$, and blood products $(2 \%)$.

- Neither antihistamine nor steroid administration in the acute phase conferred any separate beneficial effect in this series of cases.

- There was ample evidence of the beneficial effect of adrenaline administration during the acute episode. Notable was the apparent reluctance of some anaesthetists in these reports to employ adrenaline early and appropriately.

- When faced with sudden, unexpected, or severe hypotension, anaesthetists should always consider an anaphylactic or an anaphylactoid reaction.

- All patients suspected of having anaphylaxis should have plasma histamine, tryptase, and specific IGE concentrations measured at the time of the reaction and at 1 and 6 hours after the reaction.

- Of the 122 reports (including 64 "severe") where reported management was tested against the algorithms, it was considered that the properly applied structured approach would have outperformed the anaesthetist in 36 cases $(30 \%)$, including 15 of the severe reaction cases.

possible, as there is crossreactivity for up to $75 \%$ of neuromuscular blocking agents. ${ }^{15}{ }^{16}$ It should be remembered that a severe reaction can occur in response to almost any agent including colloids, dyes such as isosulfan blue which the surgeon may inject for sentinel lymph node mapping for breast cancer, ${ }^{17-18}$ and even to chlorhexidine. ${ }^{19}$

The anaphylaxis sub-algorithm described in this paper is considered a suitable, safe sub-algorithm for the management of suspected anaphylactic and anaphylactoid reactions under anaesthesia. Its widespread adoption has the potential to significantly improve and standardise current management practice. Improved documentation and patient education, follow up of all "suspicious" reactions, and letters of warning to give to future anaesthetists would also be of benefit.

\section{ACKNOWLEDGEMENTS}

The authors would like to thank all the anaesthetists in Australia and New Zealand who contributed to the 4000 incident reports upon which this and the other 24 papers in the Crisis Management Series are based. The coordinators of the project also thank Liz Brown for preparing the draft of the original Crisis Management Manual; Loretta Smyth for typing; Monika Bullock, RN, for earlier coding and classifying of data; Dr Charles Bradfield for the electronic version of the algorithms; Dr Klee Benveniste for literature research; Drs Klee Benveniste, Michal Kluger, John Williamson, and Andrew Paix for editing and checking manuscripts.

\section{Authors' affiliations}

M Currie, Clinical Quality Coordinator, Goulburn Base Hospital, Goulburn; and Consultant in Clinical Quality, Southern Area Health Service, New South Wales, Australia

R K Kerridge, Senior Staff Specialist, Department of Anaesthesia, John Hunter Hospital, Newcastle, New South Wales, Australia

A K Bacon, Consultant Anaesthetist, St John of God Hospital, Berwick, Victoria, Australia

J A Williamson, Consultant Specialist, Australian Patient Safety Foundation; Visiting Research Fellow, University of Adelaide and Royal Adelaide Hospital, Adelaide, South Australia, Australia

This study was coordinated by the Australian Patient Safety Foundation, GPO Box 400, Adelaide, South Australia, 5001, Australia.

\section{REFERENCES}

1 Mertes PM, Laxenaire MC. Allergic reactions occurring during anaesthesia. Eur J Anaesthesiol 2002;19:240-62.

2 Ryder S, Waldmann C. Anaphylaxis. Contin Educ Anaesth Crit Care Pain 2004;4:111-13.

3 Currie M, Webb RK, Williamson JA, et al. Clinical anaphylaxis: an analysis of 2000 incident reports. Anaesth Intensive Care 1993;21:621-5.

4 Runciman WB, Webb RK, Klepper ID, et al. Crisis management: validation of an algorithm by analysis of 2000 incident reports. Anaesth Intensive Care 1993;21:579-92.

5 Webb RK, Currie M, Morgan CA, et al. The Australian Incident Monitoring Study: an analysis of 2000 incident reports. Anaesth Intensive Care 1993;21:520-8.

6 Webb RK, van der Walt JH, Runciman WB, et al. Which monitor? An analysis of 2000 incident reports. Anaesth Intensive Care 1993;21:529-42.

7 Runciman WB, Kluger MT, Morris RW, et al. Crisis management during anaesthesia: the development of an anaesthetic crisis management manual. Qual Saf Health Care 2005; 14:e1

8 Fisher M. Treatment of acute anaphylaxis. BMJ 1995;311:731-3.

9 Weiss ME, Hirshman CA. Allergic drug reactions in anaesthesia, pathophysiology and management. In: Healy TEJ, Cohen PJ, eds. In:Wiley and Churchill-Davidson's A Practice of Anaesthesia, 6th edn. London: LloydLuke, 1995:516-43.

10 Fisher MM, Baldo BA. Anaphylaxis during anaesthesia: current aspects of diagnosis and prevention. Eur J Anaesthesiol 1994;11:263-84.

11 Heier T, Guttormsen AB. Anaphylactic reactions during induction of anaesthesia using rocuronium for muscle relaxation: a report including 3 cases. Acta Anaesthesiol Scand 2000;44:775-81.

12 Mertes PM, Laxenaire MC, Alla F, et al. Anaphylactic and anaphylactoid reactions occurring during anesthesia in France in 1999-2000. Anesthesiology 2003;99:536-45.

13 Naguib M, Magboul MM. Adverse effects of neuromuscular blockers and their antagonists. Middle East J Anesthesiol 1998;14:341-73.

14 Lieberman $\mathbf{P}$. Anaphylactic reactions during surgical and medical procedures. J Allergy Clin Immunol 2002; 10(Suppl 2):S64-9.

15 Mertes PM, Laxenaire MC. Adverse reactions to neuromuscular blocking agents. Curr Allergy Asthma Rep 2004;4:7-16.

16 Fisher MM, Merefield D, Baldo B. Failure to prevent an anaphylactic reaction to a second neuromuscular blocking drug during anaesthesia. $\mathrm{Br} J$ Anaesth 1999:82:770-3.

17 Montgomery LL, Thorne AC, Van Zee KJ, et al. Isosulfan blue dye reactions during sentinel lymph node mapping for breast cancer. Anesth Analg 2002;95:385-8.

18 Laurie SA, Khan DA, Gruchalla RS, et al. Anaphylaxis to isosulfan blue. Ann Allergy Asthma Immunol 2002;88:64-6.

19 Garvey LH, Roed-Petersen J, Husum B. Anaphylactic reactions in anaesthetised patients - four cases of chlorhexidine allergy. Acta Anaesthesiol Scand 2001;45:1290-4.

20 Australian Patient Safety Foundation. Crisis Management Manual: COVER $A B C D$ A SWIFT CHECK. Adelaide: Australian Patient Safety Foundation, 1996, 74 pp. Available at http://www.apsf.net.au/anaesthesia.htm (accessed 6 September 2004). 